\title{
Primary sclerosing cholangitis associated with systemic sclerosis
}

\author{
G. Fraile, J.L. Rodríguez-García and A. Moreno ${ }^{1}$ \\ Departments of Internal Medicine and ${ }^{1}$ Pathology, Hospital Ramón y Cajal, Universidad de Alcalá de \\ Henares, 28034-Madrid, Spain
}

\begin{abstract}
Summary: Primary sclerosing cholangitis is a chronic inflammatory fibrotic disorder strongly associated with inflammatory bowel disease. Although an association between some inflammatory fibrotic conditions, such as Riedel's thyroiditis and retroperitoneal fibrosis and primary sclerosing cholangitis has been described, to our knowledge there are no reports of primary sclerosing cholangitis in patients with systemic sclerosis.
\end{abstract}

A patient with this combination of conditions is presented and the possible significance of the association discussed.

\section{Introduction}

Primary sclerosing cholangitis (PSC), a chronic inflammatory disorder that ultimately results in fibrosis and obliteration of the bile ducts, is strongly associated with inflammatory bowel disease $^{1}$ and much less commonly with retroperitoneal fibrosis, regional enteritis and Riedel's thyroiditis. ${ }^{2}$ Systemic sclerosis (SS) is a multisystem disorder characterized by fibrosis that involves the skin and a variety of internal organs, although hepatic involvement in scleroderma is rare. ${ }^{3}$ We report here a patient who was found to have PSC and a year later SS. To our knowledge there are no previous reports of PSC in patients with SS although we believe that a relationship between these two fibrotic disorders might be expected on the basis of the widespread disturbance of connective tissue in SS.

\section{Case report}

A 49 year old man presented with chest pain in February 1986. Findings were a pericardial friction rub, right axillary node enlargement and hepatomegaly. Laboratory data revealed an alkaline phosphatase (ALP) level of $518 \mathrm{U} / 1$ (normal range $100-280 \mathrm{U} / \mathrm{l})$. Other biochemical tests of hepatic function were normal. Chest X-ray examination showed small bilateral pleural effusions and the

Correspondence: J.L. Rodríguez-García, M.D. Accepted: 7 August 1990 echocardiogram revealed a pericardial effusion. Immunological study yielded negative results. Axillary node biopsy specimen revealed reactive lymphadenitis. Liver biopsy showed changes most consistent with PSC: dense periductal fibrosis around septal bile ducts with atrophy of the epithelial lining cells, and mild lymphocytic infiltrate in some portal tracts (Figure 1). Endoscopic retrograde cholangiopancreatography was performed and revealed intrahepatic decreased arborization with terminal pruning of the secondary and tertiary biliary radicles (Figure 2). Upper gastrointestinal series and barium enema study were normal. PPD was positive. Urine, sputum, as well as liver, lymph node and bone marrow biopsy specimens, were negative for Mycobacterium tuberculosis. Therapy with isoniazid and rifampin was, however, prescribed for the next 9 months.

A year later the patient developed weakness and fatigue. He had been suffering Raynaud's phenomenon periodically during the previous 6 months. There were no symptoms of sicca syndrome, mouth ulcers, photosensitivity, skin rashes, dysphagia, abdominal pain, diarrhoea, arthralgias, arthritis or eye problems. The physical examination disclosed skin changes of his hands with induration, thickness and loss of hair. X-ray of the hands showed no abnormalities, and the chest X-ray film revealed no changes. There was no evidence of pericardial effusion on echocardiography. An antinuclear antibody test was positive in a dilution of $1 / 160$. The Scl-70 antibody was detected by an immunodiffusion test. Antimitochondrial antibodies were negative. Biochemical tests of liver function, including ALP, were normal. 


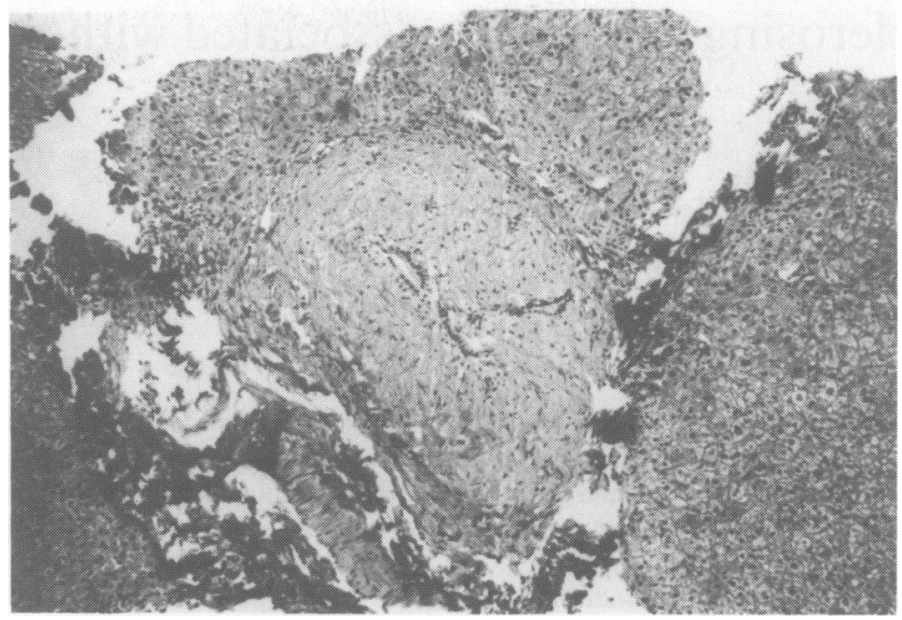

Figure 1 Photomicrograph of the liver histology showing a dense periductal fibrosis around a septal duct. The luminal : margin is irregular and the epithelial cells have an atrophic appearance (Masson $\times 120)$.

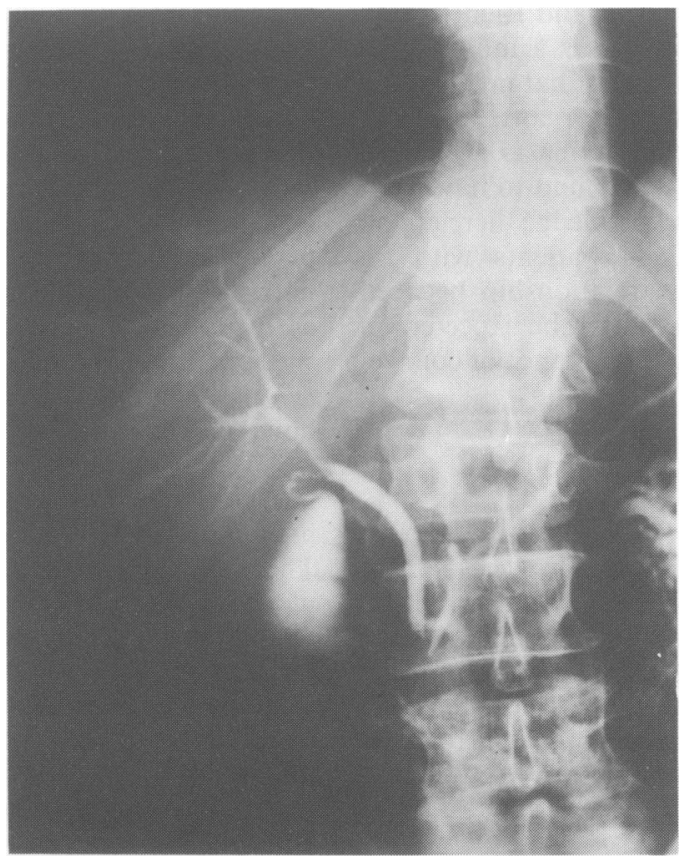

Figure 2 Endoscopic retrograde cholangiopancreatography in the same patient. The bile ducts have a narrow lumen and the intrahepatic biliary tree branching is lost. 


\section{Discussion}

Systemic sclerosis is a widespread disorder of connective tissue that involves the skin (scleroderma) and various organs of the body, including the heart, lungs, kidneys, and gastrointestinal tract. ${ }^{3}$ SS heart disease is characterized by focal myocardial necrosis and fibrosis and first of all by pericardial effusion, which is often the cause of the clinical manifestations, its incidence at post mortem examination rising until $62 \%$. The pulmonary symptoms of SS include exertional dyspnoea and cough. There is interstitial and alveolar inflammation and diffuse fibrosis in the lower two thirds of the lung. Pleural effusions are uncommon but have been mentioned together with pericardial affusions in some SS patients. ${ }^{3}$ Hepatic involvement in SS, a disease sometimes associated with primary biliary cirrhosis ${ }^{4}$ and nodular regenerative hyperplasia of the liver, ${ }^{5}$ is unusual, the pathological features being fibrosis, atrophy and inflammation of the bile ducts, commonly found on the liver biopsies of PSC patients.

Primary sclerosing cholangitis is a chronic disease with an unpredictable course, although its prognosis is considerably better than originally reported. Most patients with PSC remain asymptomatic for a considerable period of time even with cholestasic abnormalities of the liver function tests. ${ }^{6}$ Follow-up examination may demonstrate normal levels of alkaline phosphatase (ALP) ${ }^{7}$ as in our patient and some cases of PSC, confirmed by cholangiography and liver biopsy, in which the serum ALP activity is normal have been reported. ${ }^{8}$

Although the cause of both SS and PSC is largely unknown, there is evidence of immune system abnormalities and of genetic influences in these disorders. ${ }^{4,9}$ The most commonly recorded association of the major histocompatibility complex (MHC) with scleroderma is with the HLA B8, DR3-containing haplotype ${ }^{10}$ and the high fre- quency of HLA DR3 encountered in PSC patients must be noted. ${ }^{4}$ Null alleles at the MHC-linked complement component C4A locus are the most likely explanation for the increased incidence of B8 and DR3 that has been reported in certain groups of patients with SS and related disorders (vinyl chloride disease). The connection with the $\mathrm{C} 4 \mathrm{~A}$ null allele per se would suggest that a defect in the complement system may contribute to the development of SS in some patients. ${ }^{11,12}$

Immune cell-derived and other cytokines and growth factors play a critical role in regulating fibroblast proliferation. Abnormal function of the immune system in systemic sclerosis is manifested by the aberrant behaviour of connective tissue cells and by the endothelial cell damage and vasculopathy. It is not clear how antinuclear antibodies play a role in systemic sclerosis pathogenesis. One way in which antinuclear antibodies might arise is through repeated cell injury with release of nuclear constituents. ${ }^{13}$ Finally, an increased proto-oncogene (c-myc, c-myb) expression in peripheral blood $\mathrm{T}$ lymphocytes from patients with systemic sclerosis has been related with early, active disease. ${ }^{14}$ We suggest that primary sclerosing cholangitis in patients with systemic sclerosis might be the consequence of the widespread alteration of connective tissue presented in systemic sclerosis through the abnormal collagen deposition in the bile ducts epithelium.

Several reasons might explain the apparent dearth of these observations. Hepatic involvement in SS has been considered nonspecific and liver biopsy is not usually performed in patients with mild alteration of the liver function tests. Secondly, the clinical course of PSC is asymptomatic in many cases and ALP activity is normal in some patients, as indicated above. There is also a considerable overlap in the clinical and biochemical features of PSC and primary biliary cirrhosis, a disease recognized to be associated with SS. ${ }^{15}$

\section{References}

1. Aadland, E., Schrumpf, E., Fausa, O. et al. Primary sclerosing cholangitis: a long-term follow-up study. Scand J Gastroenterol 1987, 22: 655-664.

2. Bartholomew, L.G., Cain, J.C. \& Woolner, L.B. Sclerosing cholangitis: its possible association with Riedel's struma and fibrous retroperitonitis. $N$ Engl J Med 1963, 269: 8-12.

3. Rocco, V.K. \& Hurd, E.R. Scleroderma and scleroderma-like disorders. Semin Arthritis Rheum 1986, 16: 22-69.

4. Clarke, A.K., Galbraith, R.M. \& Hamilton, E.B.D. Rheumatic disorders in primary biliary cirrhosis. Ann Rheum Dis 1987, 37: 42-47.

5. Russell, M.Y. \& Kahn, H.J. Nodular regenerative hyperplasia of the liver associated with progressive systemic sclerosis: a case report with ultrastructural observation. $J$ Rheumatol 1983, 10: 748-752.

6. Schrumpf, E., Fausa, O. \& Aadland, E. Primary sclerosing cholangitis. Scand J Gastroenterol 1987, 22: 641-643.

7. Lebovics, E., Palmer, M., Woo, J. \& Schaffiner, F. Outcome of primary sclerosing cholangitis. Analysis of long-term observation of 38 patients. Arch Intern Med 1987, 147: 729-731.

8. Balasubramaniam, K., Wiesner, R.H. \& LaRusso, N.F. Primary sclerosing cholangitis with normal serum alkaline phosphatase activity. Gastroenterol 1988, 95: 1395-1398.

9. Briggs, D.C. \& Welsh, K.I. Genetic and environmental factors in scleroderma. Curr Opin Rheumatol 1989, 1: 475-478.

10. Kallenburg, C.G.M., van der Voort-Beelen, J.M. \& D'Amaro, J. Increased frequency of B8/DR3 in scleroderma and of the haplotype with impaired cellular immune response. Clin Exp Immunol 1981, 43: 478-485.

11. Briggs, D.C., Welsh, K,. Scott Pereira, R. \& Black, C.M. A strong association between null alleles at the $\mathrm{C} 4 \mathrm{~A}$ locus in the major histocompatibility complex and systemic sclerosis. Arthritis Rheum 1986, 29: 1274-1277. 
12. Briggs, D.C., Welsh, K.I., Black, C.M. \& Laurent, M.R. A strong MHC class III gene allotype association in vinyl chloride disease. Br J Rheum 1986, 25 (Suppl) Ab. 53.

13. Korn, J.H. Immunologic aspects of scleroderma. Curr Opin Rheumatol 1989, 1: 479-484.

14. Kahan, A., Gerfaux, J., Joret, A., Menkes, C.J. \& Amor, B. Increased proto-oncogene expression in peripheral blood $\mathrm{T}$ lymphocytes from patients with systemic sclerosis. Arthritis Rheum 1989, 32: 430-436.
15. Wiesner, R.H., LaRusso, N.F., Ludwing, J. \& Dickson, E.R. Comparison of the clinicopathologic features of primary biliary cirrhosis. Gastroenterology 1985, 88: 108-114. 